\title{
Quantum disordered phase on the frustrated honeycomb lattice
}

\author{
D. C. Cabra, ${ }^{1,2}$ C. A. Lamas, ${ }^{1}$ and H. D. Rosales ${ }^{1}$ \\ ${ }^{1}$ Instituto de Física de La Plata and Departamento de Física, Universidad Nacional de La Plata, C.C. 67, 1900 La Plata, Argentina \\ ${ }^{2}$ Facultad de Ingeniería, Universidad Nacional de Lomas de Zamora, Centro de Cintura y Juan XXIII, (1832) Lomas de Zamora, Argentina
}

(Received 21 September 2010; revised manuscript received 6 January 2011; published 7 March 2011)

\begin{abstract}
In the present paper we study the phase diagram of the Heisenberg model on the honeycomb lattice with antiferromagnetic interactions up to third neighbors along the line $J_{2}=J_{3}$ that includes the point $J_{2}=J_{3}=J_{1} / 2$, corresponding to the highly frustrated point where the classical ground state has macroscopic degeneracy. Using the linear spin-wave theory and the Schwinger boson technique followed by a mean field decoupling and exact diagonalization for small systems, we find an intermediate phase with a spin gap and short-range Néel correlations in the strong quantum limit $\left(S=\frac{1}{2}\right)$. All techniques provide consistent results which allow us to predict the existence of a quantum disordered phase, which may have been observed in recent high-field ESR measurements in manganites.
\end{abstract}

DOI: 10.1103/PhysRevB.83.094506

PACS number(s): 75.10.Jm, 71.10.Fd, 71.10.Hf

\section{INTRODUCTION}

The two-dimensional Heisenberg model in frustrated geometries has received a lot of attention in recent years. ${ }^{1-5}$ One of the reasons for this interest is the common belief that geometrical frustration in two-dimensional (2D) antiferromagnets may enhance the effect of quantum spin fluctuations and hence suppress magnetic order giving rise to a spin liquid. ${ }^{6}$ One candidate to test these ideas is the honeycomb lattice, which is bipartite and has a classical Néel ground state, but due to the small coordination number $(z=3)$, quantum fluctuations could be expected to be stronger than those in the square lattice and may destroy the antiferromagnetic long-range order (LRO).

The study of frustrated quantum magnets on the honeycomb lattice has also experimental motivations: On the one hand, recent ESR experiments in high magnetic field on $\mathrm{Bi}_{3} \mathrm{Mn}_{4} \mathrm{O}_{12}\left(\mathrm{NO}_{3}\right)$, which is described by a $S=3 / 2$ honeycomb Heisenberg model, have lead to the conjecture that geometric frustration plays an important role in removing the long-range magnetic order. ${ }^{7}$ On the other hand, the family of compounds $\mathrm{BaM}_{2}\left(\mathrm{XO}_{4}\right)_{2}$ with $\mathrm{M}=\mathrm{Co}$, Ni and $\mathrm{X}=\mathrm{P}$, As consists of magnetic ions $\mathrm{M}$ arranged in weakly coupled frustrated honeycomb lattices with spin $S=1 / 2$ for Co and $S=1$ for Ni. ${ }^{8}$ Short-range antiferromagnetic order below $50 \mathrm{~K}$ has been observed in another hexagonal material $\mathrm{InCu}_{2 / 3} \mathrm{~V}_{1 / 3} \mathrm{O}_{3}$, where vanadium nonmagnetic ions led to magnetic frustration. ${ }^{9}$ Finally let us mention the spin gap material $\mathrm{Na}_{3} \mathrm{Cu}_{2} \mathrm{SbO}_{6}$ whose structure is still under debate but could be described by a distorted $J_{1}-J_{3}$ hexagonal model. Last but not least, new possibilities to design interacting lattice systems with controlled geometry emerge: Modern strategies in chemistry open a route to synthesize new materials with a desired lattice structure and intersite interaction ${ }^{10}$ and the controlled setup of optical lattices for cold atoms would allow the creation of arbitrary lattice structures as well as tuning of the interactions. ${ }^{11,12}$

In the present paper we study the Heisenberg model on the honeycomb lattice with first $\left(J_{1}\right)$, second $\left(J_{2}\right)$, and third $\left(J_{3}\right)$ neighbors couplings, ${ }^{13}$ along the special line $J_{2}=J_{3}$. Using linear spin-wave theory, ${ }^{14}$ the Schwinger boson mean field theory (SBMFT), ${ }^{15}$ and exact diagonalization, we find strong evidence for the existence of an intermediate disordered region where a spin gap opens and spin-spin correlations decay exponentially. Although our results correspond to a specific line, we conjecture that the quantum disordered phase that we have found in the vicinity of the tricritical point extends within a finite region around it. Previous evidence of massive behavior in the hexagonal lattice Heisenberg model has been found in other regions of the phase space by means of exact diagonalization in Ref. 13.

\section{THE MODEL}

The Heisenberg model on the $J_{1}-J_{2}-J_{3}$ honeycomb lattice is described by

$$
H=J_{1} \sum_{N N} \overrightarrow{\mathbf{S}}_{i} \cdot \overrightarrow{\mathbf{S}}_{j}+J_{2} \sum_{N N N} \overrightarrow{\mathbf{S}}_{i} \cdot \overrightarrow{\mathbf{S}}_{j}+J_{3} \sum_{N N N N} \overrightarrow{\mathbf{S}}_{i} \cdot \overrightarrow{\mathbf{S}}_{j},
$$

where $\overrightarrow{\mathbf{S}}_{i}$ is the spin operator on site $i$. The classical model ${ }^{13,16}$ displays the different zero temperature phases shown in Fig. 1 with a tricritical point at $J_{2}=J_{3}=\frac{1}{2} J_{1}$. At this point the Hamiltonian can be written, up to a constant term, as a sum over edge-sharing plaquettes,

$$
H=\frac{J_{1}}{2} \sum_{\gamma}\left|\overrightarrow{\mathbf{S}}_{\gamma}\right|^{2},
$$

where $\overrightarrow{\mathbf{S}}_{\gamma}=\sum_{l \in \gamma} \overrightarrow{\mathbf{S}}_{l}$ is the total spin in the hexagon $\gamma$. At this particular point any state with vanishing total spin per elementary hexagon is a classical ground state, giving rise to a large GS degeneracy. This kind of situation is reminiscent of what happens in the $J_{1}-J_{2}$ square lattice case for $J_{2} / J_{1}=$ $0.5,{ }^{17,18}$ which presents a disordered spin gapped phase around this point at the quantum level.

Motivated by this analogy, we study here the region around the tricritical point $J_{2}=J_{3}=\frac{1}{2} J_{1}$ keeping $J_{2}=J_{3}$ as shown in Fig. 1. We construct in the following the corresponding magnetic phase diagram with the results obtained by means of linear spin-wave theory, the Schwinger boson technique, and exact diagonalization. 


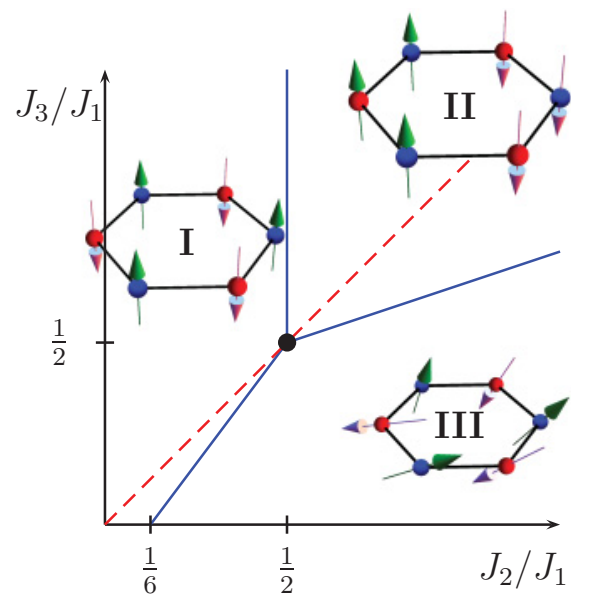

FIG. 1. (Color online) Classical phase diagram for model 1. There are three different phases schematized by the cartoons: the Néel phase (I), which has two (antiparallel) ferromagnetic triangular sublattices (blue and red balls), the collinear phase (II) where ferromagnetic chains are antiparallel, and the spiral phase (III). We focus on the dashed line corresponding to $J_{2}=J_{3}$ that includes the special point $\left(J_{2}=J_{3}=J_{1} / 2\right)$ where the ground state is infinitely degenerate.

\section{CLASSICAL PHASES AND QUANTUM CORRECTIONS VIA LINEAR SPIN-WAVE THEORY}

The classical phase diagram reduces to that shown in the line $1 / S_{c}=0$ of Fig. 2 where two collinear phases meet at the classical critical point $J_{2} / J_{1}=0.5$. A general ordered planar spin configuration can be written as

$$
\overrightarrow{\mathbf{S}}_{\vec{R}, \alpha}=S\left(\cos \left(\vec{Q} \cdot \vec{R}+\phi_{\alpha}\right) \check{e}_{1}+\sin \left(\vec{Q} \cdot \vec{R}+\phi_{\alpha}\right) \check{e}_{2}\right),
$$

where $\vec{R}$ is the position of the unit cell and $\check{e}_{1}, \check{e}_{2}$ are the primitive vectors of the direct lattice. The magnetic configuration is characterized by the ordering wave vector $\vec{Q}$ and the internal phase $\phi_{\alpha}$, where $\alpha=A, B$ is an internal index on each sublattice. There are two different phases for the classical ground state: for $J_{2} / J_{1}<0.5$ the ground state corresponds to the Néel phase, with ordering vector $\vec{Q}=(0,0)$ and $\phi_{A}-\phi_{B}=\pi$, and for $J_{2} / J_{1}>0.5$ the ground state corresponds to a collinear phase with $\vec{Q}=(2 \pi / \sqrt{3}, 0)$ and $\phi_{A}-\phi_{B}=\pi$. In the following we call this phase the columnar antiferromagnetic (CAF) phase.

Using linear spin-wave theory (LSWT) ${ }^{14,19}$ we have investigated the stability of the classical phase diagram. In this theory the spin operators are expressed by bosonic operators using the standard Holstein-Primakoff representation which to lowest order in $1 / S$ reads

$$
\begin{aligned}
\mathbf{S}_{\vec{x}}^{z} & =S-\mathbf{a}_{\vec{x}}^{\dagger} \mathbf{a}_{\vec{x}}, \\
\mathbf{S}_{\vec{x}}^{+} & =\sqrt{2 S} \mathbf{a}_{\vec{x}}, \\
\mathbf{S}_{\vec{x}}^{-} & =\sqrt{2 S} \mathbf{a}_{\vec{x}}^{\dagger} .
\end{aligned}
$$

The Hamiltonian (1) is written in terms of these bosonic operators and then Fourier transformed. As the Hamiltonian contains only quadratic operators it can be diagonalized numerically by means of a para-unitary transformation. ${ }^{20}$ The order parameter in the Néel phase corresponds to the staggered

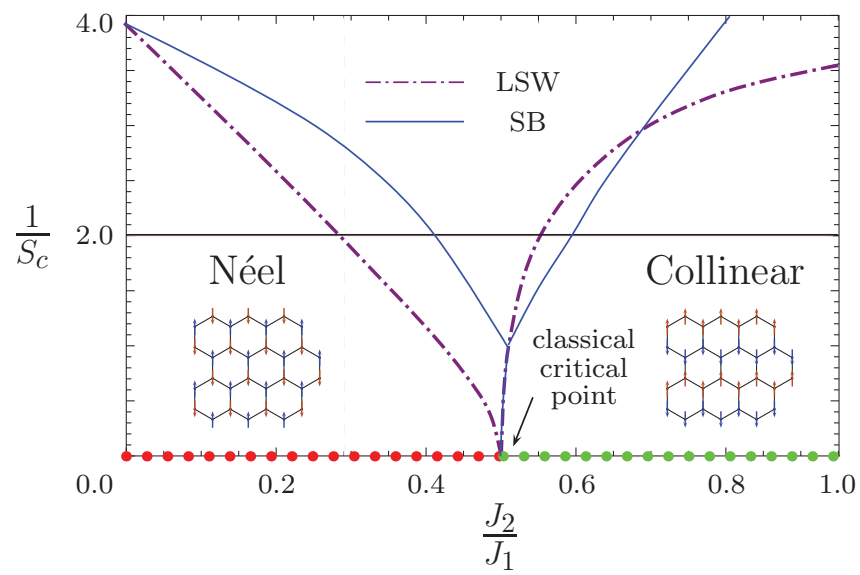

FIG. 2. (Color online) Inverse of the critical spin $S_{c}$ as a function of $J_{2}$. The dashed-point line shows the bound obtained by LSWT. Above this line, quantum fluctuations destroy the magnetic order, and the staggered magnetization goes to zero. For $S=1 / 2$ (full black line), there is a small range $0.29<J_{2} / J_{1}<0.55$ where there is not magnetic order; the full blue line corresponds to the edge obtained by SBMFT. For the $S=1 / 2$ case, there is a range $0.41<J_{2} / J_{1}<0.6$ where the system has a spin-gap indicating a quantum disordered phase (see Fig. 4). The dotted line correspond to the classical limit $S \rightarrow \infty$ where the ground state corresponds to the Néel phase in the region $J_{2} / J_{1}<0.5$, while for $J_{2} / J_{1}>0.5$ the ground state corresponds to the $\mathrm{CAF}$ phase.

magnetization, while in the collinear phase it corresponds to the magnetization in the zigzag direction and staggered magnetization in the armchair direction. The results obtained are summarized in Fig. 2 as a function of the spin $S$ and the frustration $\left(J_{2} / J_{1}\right)$ (we do not present details of the different steps of LSWT since these are standard $\left.{ }^{14,21,22}\right)$. The edge of the ordered phases was obtained by finding the frustration $\left(J_{2} / J_{1}\right)$ at which the quantum fluctuations destroy the classical order, i.e., where the order parameter vanishes. The results show that quantum fluctuations reduce the stability of the Néel phase around the classical point $J_{2} / J_{1}=1 / 2$ : for $S=1 / 2$ Néel order is found for $0<J_{2} / J_{1}<0.29$ and CAF order for $0.55<J_{2} / J_{1}$.

An improvement of LSWT was introduced by Takahashi in Ref. 23 where an extra constraint of zero sublattice magnetization is introduced and the equations are solved in a self-consistent way. This modified LSWT has led to results that compare well with SBMFT results in different lattices. It would then be interesting to apply this method to the present system to compare with the results we present below. However this is out of the scope of the present paper.

To further analyze the region $0.29<J_{2} / J_{1}<0.55$ which is seen as a nonmagnetic phase within LSWT we now use SBMFT and exact diagonalization.

\section{SCHWINGER BOSON MEAN FIELD THEORY AND EXACT DIAGONALIZATION}

The Heisenberg model on the honeycomb lattice was studied using SBMFT by Mattsson et al. ${ }^{24}$ for antiferromagnetic interactions at first and second neighbors. Here we study 
Hamiltonian (1) using a rotationally invariant version of this technique, which has proven successful in incorporating quantum fluctuations. ${ }^{25-27}$

In the Schwinger boson approach, the Heisenberg interaction is written as a biquadratic form. In this representation the spin operators on each site are replaced by two species of bosons via the relation

$$
\overrightarrow{\mathbf{S}}_{\vec{x}}=\frac{1}{2} \overrightarrow{\mathbf{b}}_{\vec{x}}^{\dagger} \cdot \vec{\sigma} \cdot \overrightarrow{\mathbf{b}}_{\vec{x}}
$$

where $\overrightarrow{\mathbf{b}}_{\vec{x}}^{\dagger}=\left(\mathbf{b}_{\vec{x} \uparrow}^{\dagger}, \mathbf{b}_{\vec{x} \downarrow}\right)$ is a bosonic spinor, $\vec{\sigma}$ is the vector of Pauli matrices, and there is a boson-number restriction $\sum_{\sigma} \mathbf{b}_{\vec{x} \sigma}^{\dagger} \mathbf{b}_{\vec{x} \sigma}=2 S$ on each site. With this representation, the rotationally invariant spin-spin interaction can be written as

$$
\overrightarrow{\mathbf{S}}_{\vec{x}} \cdot \overrightarrow{\mathbf{S}}_{\vec{y}}=: \mathbf{B}_{\vec{x}, \vec{y}}^{\dagger} \mathbf{B}_{\vec{x}, \vec{y}}:-\mathbf{A}_{\vec{x}, \vec{y}}^{\dagger} \mathbf{A}_{\vec{x}, \vec{y}},
$$

where $\sigma= \pm,: \mathbf{O}$ : indicates the normal ordering of the operator $\mathbf{O}$, and we define the $\mathbf{S U}(2)$ invariants

$$
\begin{aligned}
\mathbf{A}_{\vec{x}, \vec{y}} & =\frac{1}{2} \sum_{\sigma} \sigma \mathbf{b}_{\vec{x}, \sigma} \mathbf{b}_{\vec{y},-\sigma}, \\
\mathbf{B}_{\vec{x}, \vec{y}}^{\dagger} & =\frac{1}{2} \sum_{\sigma} \mathbf{b}_{\vec{x}, \sigma}^{\dagger} \mathbf{b}_{\vec{y}, \sigma} .
\end{aligned}
$$

The operator $\mathbf{A}_{\vec{x}, \vec{y}}$ creates a spin singlet pair between sites $\vec{x}$ and $\vec{y}$ and $\mathbf{B}_{\vec{x}, \vec{y}}$ creates a ferromagnetic bond, which implies the intersite coherent hopping of the Schwinger bosons. This rotationally invariant decoupling, which enables us to treat ferromagnetism and antiferromagnetism on equal footing, was introduced by Ceccato et al. ${ }^{25}$ Later, Flint and Coleman ${ }^{26}$ presented a generalization to large $N$ that shows substantial improvements over the $\operatorname{SP}(N)$ approach.

To construct a mean field theory, we perform the HartreeFock decoupling

$$
\begin{aligned}
\left(\overrightarrow{\mathbf{S}}_{\vec{x}+\vec{r}_{\alpha}} \cdot \overrightarrow{\mathbf{S}}_{\vec{y}+\vec{r}_{\beta}}\right)_{\mathrm{MF}}= & {\left[B_{\alpha \beta}^{*}(\vec{x}-\vec{y}) \mathbf{B}_{\vec{x}+\vec{r}_{\alpha}, \vec{y}+\vec{r}_{\beta}}\right.} \\
& -A_{\alpha \beta}^{*}(\vec{x}-\vec{y}) \mathbf{A}_{\left.\vec{x}+\vec{r}_{\alpha}, \vec{y}+\vec{r}_{\beta}+\text { h.c. }\right]} \\
& -\left\langle\left(\overrightarrow{\mathbf{S}}_{\vec{x}+\vec{r}_{\alpha}} \cdot \overrightarrow{\mathbf{S}}_{\vec{y}+\vec{r}_{\beta}}\right)_{\mathrm{MF}}\right\rangle,
\end{aligned}
$$

where

$$
\begin{gathered}
A_{\alpha \beta}^{*}(\vec{x}-\vec{y})=\left\langle\mathbf{A}_{\vec{x}+\vec{r}_{\alpha}, \vec{y}+\vec{r}_{\beta}}^{\dagger}\right\rangle, \\
B_{\alpha \beta}^{*}(\vec{x}-\vec{y})=\left\langle\mathbf{B}_{\vec{x}+\vec{r}_{\alpha}, \vec{y}+\vec{r}_{\beta}}^{\dagger}\right\rangle, \\
\left\langle\left(\overrightarrow{\mathbf{S}}_{\vec{x}+\vec{r}_{\alpha}} \cdot \overrightarrow{\mathbf{S}}_{\vec{y}+\vec{r}_{\beta}}\right)_{\mathrm{MF}}\right\rangle=\left|B_{\alpha \beta}(\vec{x}-\vec{y})\right|^{2}-\left|A_{\alpha \beta}(\vec{x}-\vec{y})\right|^{2} .
\end{gathered}
$$

These are the mean field equations and must be solved in a self-consistent way together with the constraints on the number of bosons

$$
B_{\alpha \alpha}(R=0)=2 N_{c} S,
$$

where $N_{c}$ is the total number of unit cells and $S$ is the spin strength. Finding numerical solutions of Eqs. (9) and (11) involves finding the roots of 24 coupled nonlinear equations for the parameters $A$ and $B$ plus the additional constraints to determine the values of the Lagrange multipliers $\lambda^{(\alpha)}$. We perform the calculations for finite but very large lattices and finally we extrapolate the results to the thermodynamic limit. We solve numerically Eqs. (9) and (11) for several values of the frustration parameter $J_{2} / J_{1}$ and with the values obtained for the MF parameters and the Lagrange multipliers we compute the energy and the new values for the MF parameters. We repeat this self-consistent procedure until the energy and the MF parameters converge. After reaching convergence we can compute all physical quantities such as the energy, the spinspin correlations, and the excitation gap. In order to support the analytical results of the MF approach, we have also performed exact diagonalization on finite systems with 18,24 , and 32 spins with periodic boundary conditions for $S=1 / 2$ using SPINPACK. ${ }^{28}$

In Fig. 3(a) we show the ground state energy per unit cell as a function of the frustration for a system of 32 sites calculated by means of SBMFT and ED, showing an excellent agreement between both approaches. The advantage of the SBMFT is that it allows us to study much larger systems: We have studied different system sizes up to 3200 sites, which we extrapolate to the thermodynamic limit.
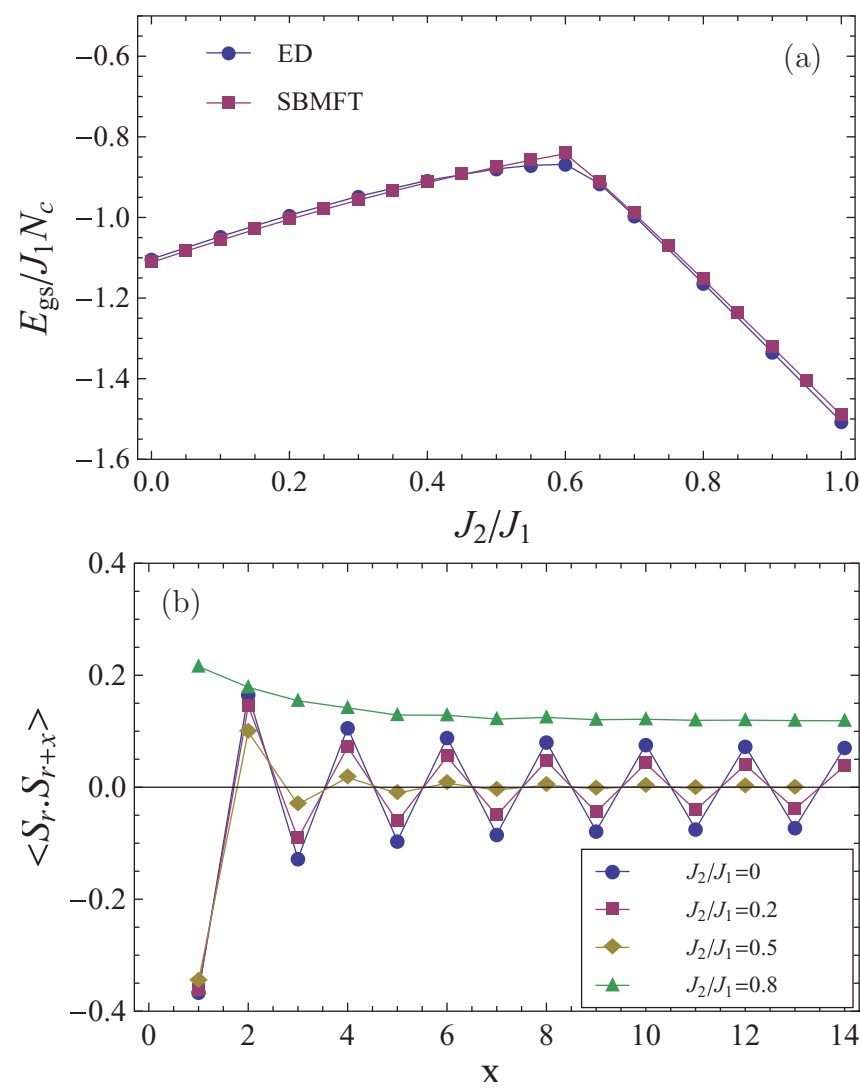

FIG. 3. (Color online) (a) GS energy per unit cell as a function of $J_{2} / J_{1}$ for a lattice of 32 sites. The circles are exact results (ED) and the squares are the SBMFT results. (b) Spin-spin correlation function (SSCF) vs distance $X$ in the zigzag direction obtained within SBMFT. For $0<J_{2} / J_{1}<0.41$, the SSCF corresponds to the Néel phase with long-rage order (LRO); for $0.41<J_{2} / J_{1}<0.6$ the correlations are short range, indicating a gap zone with shortrange order (SRO); and for $0.6<J_{2} / J_{1}$ the correlations correspond to the collinear phase (ferromagnetic correlations in the zigzag direction). 


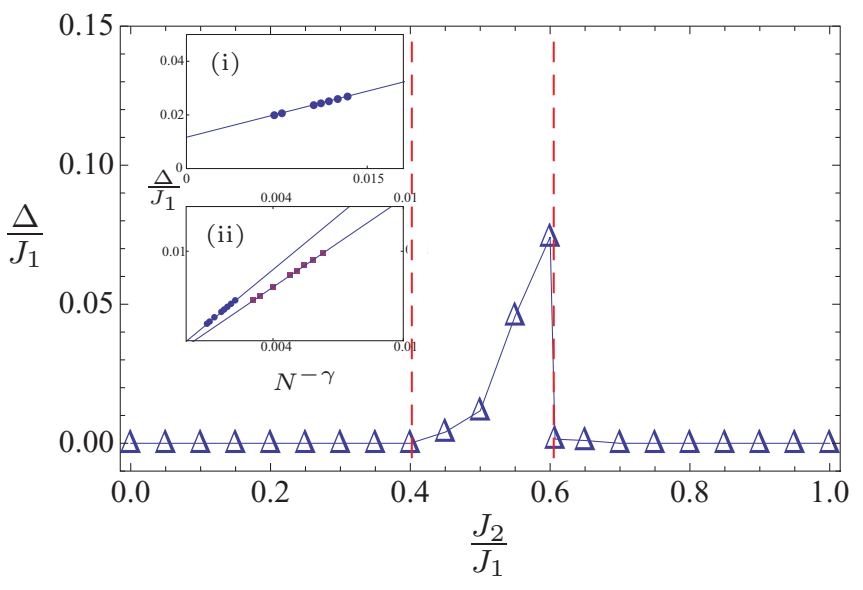

FIG. 4. (Color online) Gap in the boson dispersion as a function of $J_{2} / J_{1}$ for $S=1 / 2$. In the region $J_{2} / J_{1} \sim 0.6$ the system remains gapped. Inset: finite size scaling for the gap. (i) $J_{2} / J_{1}=0.5$ $(\gamma=0.6451)$. (ii) Circles correspond to $J_{2} / J_{1}=0.05(\gamma=0.911)$ and squares correspond to $J_{2} / J_{1}=0.35(\gamma=0.758)$.

For the present model we only find commensurate collinear phases, and for these phases the wave vector $Q_{0}$, where the dispersion relation has a minimum, remains pinned at a commensurate point in the Brillouin zone, independently of the value of the frustration $J_{2} / J_{1}$. In the thermodynamic limit, a state with LRO is characterized in the Schwinger boson approach by a condensation of bosons at the wave vector $Q_{0}$. This implies that the dispersion of the bosons in a state with LRO is gapless. As we discussed earlier, we solve (9) and (11) for finite systems, then to detect LRO we calculate the gap in the bosonic spectrum as a function of $J_{2} / J_{1}$ for different system sizes and perform a finite size scaling, finding a finite region where the system remains gapped.

The extrapolation of the boson gap as a function of the frustration is shown in Fig. 4. In the range of $0.41<J_{2} / J_{1}<0.6$ the system presents a finite gap. The inset shows an example of the finite size scaling for different values of the frustration. The structure of the different phases can be understood calculating the spin-spin correlation function (SSCF).
For $J_{2} / J_{1}<0.41$ the SSCF is antiferromagnetic in all directions and is long range while for $0.6<J_{2} / J_{1}$ we have found ferromagnetic LRO correlations in the zigzag direction that correspond to the CAF phase. The most interesting result is in the intermediate region $0.41<J_{2} / J_{1}<0.6$ where the results for the SSCF are consistent with the presence of a gap. In this region we have found short-range antiferromagnetic correlations. A plot of the SSCF for $J_{2} / J_{1}=0,0.2,0.5$, and 0.8 obtained within SBMFT is presented in Fig. 3(b). Figure 2 shows the ground state phase diagram as a function of $1 / S$. On the one hand, for $1 / S$ smaller than a critical value $1 / S_{c}\left(J_{2} / J_{1}\right)$, the correlation function has LRO, characterized by a condensation of bosons at the wave vector $Q_{0}$. On the other hand, when $1 / S$ is greater than $1 / S_{c}\left(J_{2} / J_{1}\right)$, the correlation functions have $\mathrm{SRO}$, indicating quantum disorder.

\section{CONCLUSIONS}

In summary, the results obtained with the different techniques used in the present paper suggest the existence of a region in the intermediate frustration regime where the system does not show quantum magnetic order for $S=1 / 2$. On the one hand, LSWT analysis predicts that the Néel LRO region extends up to $J_{2} / J_{1} \approx 0.29$ and the collinear antiferromagnet LRO is present for $J_{2} / J_{1}>0.55$. In the intermediate region we find no evidence of any type of magnetic order with this technique. On the other hand the results found with SBMFT predict a quantum disordered region $0.41<J_{2} / J_{1}<0.6$. In this region a gap opens in the bosonic dispersion and the spin-spin correlation function shows Néel short-range order followed by the LRO CAF phase for $J_{2} / J_{1}>0.6$.

\section{ACKNOWLEDGMENTS}

We would like to thank G. L. Rossini, A. E. Trumper, and L. O. Manuel for helpful discussions and P. Pujol for pointing out to us an error in a previous version. This work was partially supported by ESF Grant INSTANS, PICT ANPCYT (Grant No 20350) and PIP CONICET (Grant No 1691).
${ }^{1}$ M. A. Metlitski and S. Sachdev, Phys. Rev. B 77, 054411 (2008).

${ }^{2}$ R. K. Kaul, M. A. Metlitski, S. Sachdev, and C. Xu, Phys. Rev. B 78, 045110 (2008).

${ }^{3}$ L. Wang and A. W. Sandvik, Phys. Rev. B 81, 054417 (2010).

${ }^{4}$ R. Moessner, S. L. Sondhi, and P. Chandra, Phys. Rev. B 64, 144416 (2001).

${ }^{5}$ A. Ralko, M. Mambrini, and D. Poilblanc, Phys. Rev. B 80, 184427 (2009).

${ }^{6}$ P. W. Anderson, Science 235, 1196 (1987).

${ }^{7}$ S. Okubo, F. Elmasry, W. Zhang, M. Fujisawa, T. Sakurai, H. Ohta, M. Azuma, O. A. Sumirnova, and N. Kumada, J. Phys. Conf. Ser. 200, 022042 (2010).
${ }^{8}$ Magnetic Properties of Layered Transition Metal Compounds, edited by L. J. De Jongh (Kluwer, Dordrecht, 1990).

${ }^{9}$ A. Möller, U. Löw, T. Taetz, M. Kriener, G. André, F. Damay, O. Heyer, M. Braden, and J. A. Mydosh, Phys. Rev. B 78, 024420 (2008).

${ }^{10}$ R. Arita, Y. Suwa, K. Kuroki, and H. Aoki, Phys. Rev. Lett. 88, 127202 (2002); Y. Suwa, R. Arita, K. Kuroki, and H. Aoki, Phys. Rev. B 68, 174419 (2003); A. Harrison, J. Phys. Condens. Matter 16, S553 (2004); Y. Z. Zheng, M. L. Tong, W. Xue, W. X. Zhang, X. M. Chen, F. Grandjean, and G. J. Long, Angew. Chem. Int. Ed. 46, 6076 (2007).

${ }^{11}$ D. Jaksch and P. Zoller, Ann. Phys. (NY) 315, 52 (2005). 
${ }^{12}$ M. Lewenstein, A. Sanpera, V. Ahufinger, B. Damski, A. Sen De, and U. Sen, Adv. Phys. 56, 243 (2007).

${ }^{13}$ J. B. Fouet, P. Sindzingre, and C. Lhuillier, Eur. Phys. J. B 20, 241 (2001).

${ }^{14} \mathrm{~A}$. Auerbach, Interacting Electrons and Quantum Magnetism (Springer-Verlag, New York, 1994).

${ }^{15}$ A. Auerbach and D. P. Arovas, in Introduction to Frustrated Magnetism, edited by C. Lacroix, P. Mendels, and F. Mila (Springer, New York, 2011).

${ }^{16}$ E. Rastelli, A. Tassi, and L. Reatto, Physica B 97, 1 (1979).

${ }^{17}$ P. Chandra and B. Doucot, Phys. Rev. B 38, 9335 (1988); E. Dagotto and A. Moreo, Phys. Rev. Lett. 63, 2148 (1989); N. Read and S. Sachdev, ibid. 66, 1773 (1991); H. J. Schulz and T. A. L. Ziman, Europhys. Lett. 18, 355 (1992); F. Figueirido, A. Karlhede, S. Kivelson, S. Sondhi, M. Rocek, and D. S. Rokhsar, Phys. Rev. B 41, 4619 (1990); M. E. Zhitomirsky and K. Ueda, ibid. 54, 9007 (1996); R. R. P. Singh, Z. Weihong, C. J. Hamer, and J. Oitmaa, ibid. 60, 7278 (1999); V. N. Kotov, J. Oitmaa, O. P. Sushkov, and Z. Weihong, ibid. 60, 14613 (1999); L. Capriotti and S. Sorella, Phys. Rev. Lett. 84, 3173 (2000); J. Sirker, Z. Weihong, O. P. Sushkov, and J. Oitmaa, Phys. Rev. B 73, 184420 (2006); R. Darradi, O. Derzhko, R. Zinke, J. Schulenburg, S. E. Kruger, and
J. Richter, ibid. 78, 214415 (2008); J. Reuther and P. Wölfle, ibid. 81, 144410 (2010).

${ }^{18}$ J. Richter and J. Schulenburg, Eur. Phys. J. B 73, 117 (2010).

${ }^{19}$ The $J_{1}-J_{2}-J_{3}$ Heisenberg model on the honeycomb lattice was analyzed previously using LSWT in Ref. 13, but the intermediate phase along the line $J_{2}=J_{3}$, which is the main aim of the present paper, was not analyzed in detail.

${ }^{20}$ J. H. P. Colpa, Physica A 93, 327 (1978).

${ }^{21}$ A. Altland and B. Simons, Condensed Matter Field Theory (Cambridge University Press, Cambridge, 2006).

${ }^{22}$ A. E. Trumper, L. Capriotti, and S. Sorella, Phys. Rev. B 61, 11529 (2000).

${ }^{23}$ M. Takahashi, Phys. Rev. B 40, 2494 (1989).

${ }^{24}$ A. Mattsson, P. Frojdh, and T. Einarsson, Phys. Rev. B 49, 3997 (1994).

${ }^{25}$ H. A. Ceccatto, C. J. Gazza, and A. E. Trumper, Phys. Rev. B 47, 12329 (1993).

${ }^{26}$ R. Flint and P. Coleman, Phys. Rev. B 79, 014424 (2009).

${ }^{27}$ A. E. Trumper, L. O. Manuel, C. J. Gazza, and H. A. Ceccatto, Phys. Rev. Lett. 78, 2216 (1997).

${ }^{28}$ J. Schulenburg, program package SPINPACK [http://www-e.unimagdeburg.de/jschulen/spin/]. 